\title{
Albert Camus précurseur. Méditerranée d'hier et d'aujourd'hui, textes réunis et présentés par Alek Baylee Toumi
}

\section{Gian Luigi Di Bernardini}

\section{(2) OpenEdition \\ Journals}

\section{Edizione digitale}

URL: http://journals.openedition.org/studifrancesi/6086

DOI: $10.4000 /$ studifrancesi.6086

ISSN: 2421-5856

\section{Editore}

Rosenberg \& Sellier

\section{Edizione cartacea}

Data di pubblicazione: 1 mai 2011

Paginazione: 209-210

ISSN: 0039-2944

\section{Notizia bibliografica digitale}

Gian Luigi Di Bernardini, «Albert Camus précurseur. Méditerranée d'hier et d'aujourd'hui, textes réunis et présentés par Alek Baylee Toumi», Studi Francesi [Online], 163 (LV | I) | 2011, online dal 30 novembre 2015, consultato il 10 janvier 2021. URL: http://journals.openedition.org/studifrancesi/6086 ; DOI: https://doi.org/10.4000/studifrancesi.6086

Questo documento è stato generato automaticamente il 10 janvier 2021.

\section{cc) (†) $\odot$}

Studi Francesi è distribuita con Licenza Creative Commons Attribuzione - Non commerciale - Non opere derivate 4.0 Internazionale. 


\title{
Albert Camus précurseur. Méditerranée d'hier et d'aujourd'hui, textes réunis et présentés par Alek Baylee Toumi
}

\author{
Gian Luigi Di Bernardini
}

\section{NOTIZIA}

Albert Camus précurseur. Méditerranée d'hier et d'aujourd'hui, textes réunis et présentés par Alek BAYLEE TOUMI, New York, Peter Lang, 2009 («Franco-phone Cultures \& Literatures», 55), pp. 161.

1 Albert Camus précurseur era il titolo di un convegno, tenutosi presso l'Università del Wisconsin a Madison tra il 22 e il 23 settembre 2006, di cui questo volume raccoglie gli atti; i due temi costitutivi: «Camus, lecteur de l'actuel» e "Camus et la Méditerranée» non generano, tuttavia, delle sezioni separate nella versione scritta ed è per questo che la sintesi degli interventi segue il loro ordine di presentazione.

2 Amina AZZA-BEKKAT, in Albert Camus et l'Algérie: le malentendu (pp.9-21), offre una ricostruzione generale dell'itinerario dell'autore che permette di mettere a fuoco le complesse ragioni alla base di quell'ostracismo a cui l'opera di Camus è andata incontro. Hélène DIAZ BROWN, in Le Désert, agent révélateur de l'oppression coloniale dans "L'Hôte" et "La Femme adultère" d'Albert Camus (pp. 23-36), lavora sui protagonisti di due novelle de L'Exil et le royaume e sul loro rapporto col deserto, la cui bellezza genera un desiderio di appartenenza che viene, però, costantemente frustrato proprio a causa della menzogna coloniale, la quale perverte per sua natura le nozioni di possesso e appartenenza. Ali YEDES (Identités coloniales et métropolitaines dans l'œeuvre de Camus, pp. 37-43) sottolinea il doppio statuto identitario di Camus, che si sentiva estraneo alla propria terra, ma anche esiliato in Francia. Bernard ARESU (Albert Camus, EuropeanAlgerian Writing, and the Aporia of Identity, pp. 45-60) rilancia il tema dell'identità ibrida dell'autore ponendo tuttavia l'accento sul suo versante politico, che il critico studia 
attraverso alcuni documenti preparatori de Le Premier homme. Hélène RUFAT (De la rêverie méditerranéenne à l'esprit libertaire: présence de l'Espagne dans l'oeuvre camusienne, pp.61-71) esplora i tratti specifici della presenza di una sorta di "mito mediterraneo" nella produzione dell'autore, saldamente collegato alla nozione di libertarismo di cui gli spagnoli sarebbero storicamente portatori. Raymond GAY-CROSIER (Camus, Our Familiar Stranger, pp. 73-88) lavora in una prima parte del suo intervento su L'Étranger per chiarire la sensazione di "stranezza" che a volte l'opera e la figura di Camus producono; in una seconda parte il critico concentra la sua attenzione su L'Homme revolté, o meglio su un articolo in sua difesa scritto a metà anni '50. Jan RIGAUD (Albert Camus, ou l'envers des noces méditerranéennes, pp. 89-101) torna sul tema dell'identità di Camus, la quale, eterogenea per ascendenza biologica, trova nella nozione di mediterraneo un sistema ancorché costruito - per armonizzare elementi originariamente discordanti. David R. ELLISON (La Pensée/le partage de midi: le dernier Camus et la Méditerranée, pp.105-115) prosegue l'interrogazione sull'identità camusiana approfondendo il suo pensiero degli anni ' 50 e identificando due poli nel pensiero dello scrittore, l'uno relativo alla nozione di condivisione del Mediterraneo tra i popoli che vi si affacciano, l'altro inerente ad un'esclusività di tale ricchezza rispetto a tutti i popoli che non vi si affacciano. Denise BRAHMI (Révolte, révolution, terreur, pp.117-125) analizza l'uso che Camus fa dei tre termini annunciati dal titolo in un corpus di scritti pubblicati tra la fine della Seconda Guerra Mondiale e l'inizio della guerra d'Algeria. Janice Gross (Albert Camus and Contemporary Algerian Playwrights: a Shared Faith in Dialogue, pp. 127-140) si interessa della nuova generazione di drammaturghi algerini che sembrano finalmente attingere all'eredità camusiana. Infine Ralph schoolCRAFT (The Renegade: Camus or Sartre, pp. 141-156) studia la polemica Sartre-Camus attraverso la novella Le Renégat. 